\title{
LA ARGUMENTACIÓN EN LA COMUNIDAD CIENTÍFICA Y EN LA FORMACIÓN DE PROFESORES DE FÍSICA
}

\author{
Argumentation in scientific community \\ and in Physics teachers' education
}

\author{
Stella Maris Islas ${ }^{1}$ \\ Margarita Rosa Sgro ${ }^{2}$ \\ Marta Azucena Pesa ${ }^{3}$
}

Resumen: Se informa una parte de una investigación sobre la formación epistemológica de profesores de Física, que apunta a ofrecer fundamentos y orientaciones para mejorar esa formación. Asumiendo que el conocimiento de algunas particularidades de la comunicación entre científicos puede contribuir a desestabilizar las concepciones erróneas de la ciencia que frecuentemente subyacen a las acciones docentes, se presentan en la primera sección del artículo ciertas referencias y ejemplos de argumentaciones sostenidas entre científicos. Se aboga por una formación docente que contemple las argumentaciones que se dan en la ciencia, como aporte para la construcción de visiones menos estereotipadas del razonamiento científico y para el reconocimiento del carácter social de la generación de conocimiento en el ámbito de la ciencia. La información recogida sobre contenidos de Epistemología en la formación docente permite vislumbrar que es posible incorporar estas temáticas, lo cual debería ser acompañado con algunas renovaciones en la dinámica de los cursos de formación.

Palabras clave: Argumentación. Formación epistemológica de docentes.

Abstract: This paper reports part of a research project on the epistemological education of Physics teachers, aimed at offering foundations and guidelines to improve that education. It assumes that appreciating some particularities of the communication between scientists could contribute to challenging erroneous conceptions of science which frequently underlie educational actions. The first section of this article presents some examples of argumentation maintained between scientists. It endorses teacher training that gives relevance to discussions within science as a contribution to building less stereotyped visions of scientific reasoning and to realizing the social character of knowledge construction in science. Collected data on epistemological content in teacher education allows us to suppose that is possible to incorporate these themes. Some changes in training course dynamics would be associated with it.

Keywords: Argumentation. Teachers' education in epistemology.

${ }^{1}$ Física. Doctoranda en Ciencias de la Educación. Docente, Departamento de Formación Docente, Facultad de Ciencias Exactas, Universidad Nacional del Centro de la Provincia de Buenos Aires. Argentina.

<sislas@exa.unicen.edu.ar>

${ }^{2}$ Licenciada en Ciencias de la Educación. Doctora en Educación. Docente, área de Pedagogía, Facultad de Ciencias Humanas, Universidad Nacional del Centro. Buenos Aires, Argentina.<msgro@ffch.unicen.edu.ar>

${ }^{3}$ Física. Doctora en Física. Docente, Departamento de Física, Facultad de Ciencias Exactas y Tecnología, Universidad Nacional de Tucumán, Argentina. <mpesa@rectorado.unt.edu.ar>

${ }^{1}$ Fiori, 1291

Tandil (7000)

Argentina 
Islas, S. M.; Sgro, M. R.; Pesa, M. A.

\section{Introducción}

Este artículo recoge algunos de los resultados alcanzados en una investigación sobre la formación epistemológica de los profesores de Física. Se focaliza en los saberes docentes acerca del carácter dialógico de la construcción del conocimiento científico. Como punto de partida, se toma información ya existente sobre deficiencias de la formación docente en cuestiones epistemológicas, teniendo como meta el aportar fundamentos de investigación para orientar propuestas de ciertos cambios en la formación de profesores de Física en la Argentina. Tales cambios promoverían en los docentes una tendencia favorable a conferir un sesgo más dialógico a sus clases.

Pueden reconocerse dos líneas en este trabajo: la formación docente en Física, y las formas de comunicación entre investigadores en esa disciplina. Se cuenta con un cuerpo de antecedentes de investigación en la primera de ellas, siendo más escasos los datos de campo y los consensos teóricos en referencia a la segunda temática. Con este artículo, además de mostrar la vinculación entre las dos líneas, se procura realizar un aporte a la discusión sobre argumentaciones en la comunidad de investigadores en educación científica.

Se comienza con uno de los capítulos de la investigación: el que se propone ofrecer evidencias del empleo, en la investigación en Física, de inferencias cuya validación no deviene de la sintaxis de las mismas. La hipótesis respecto de la posibilidad de encontrarlas en el ámbito de la Física surgió de analizar resultados obtenidos por otros investigadores (DUNBAR, 2000). Se han hecho registros de la presencia de analogías y abducciones en algunos de los debates que se editan en la literatura científica. Esta temática ha sido elegida porque - de acuerdo con los datos que ofrece la investigación sobre concepciones de ciencia en los docentes - uno de los componentes de la visión ingenua de la ciencia que se encuentra con frecuencia en el ámbito docente es la creencia en su carácter cuasi-dogmático, fundada en parte en suponer la no existencia de dudas y disensos y, también, en la presunción de que los razonamientos científicos solamente pueden ser: inductivos (visión empirista de la ciencia) o bien deductivos, es decir, con resultados garantizados por la lógica.

Sobre la base de los resultados que se enuncian en la primera sección del artículo, la segunda intenta mostrar que es posible generar situaciones que ayuden a los docentes a percibir y valorar este aspecto de la dinámica de producción de novedades científicas. Mediante algunas modificaciones en los contenidos de Epistemología que se abordan en la formación docente e implementando ciertas estrategias (discursivas, argumentativas) en las clases destinadas a docentes, ellos podrían estar en mejores condiciones para reconstruir sus concepciones, y superar las visiones ingenuas de la actividad científica. En concordancia con ello, en lugar de presentar al contenido científico de sus clases como mera información condensada en una serie de sentencias inmutables que los alumnos han de memorizar, los docentes serían más proclives a poner en juego estrategias conducentes a la construcción de conocimiento más creativas y variadas que las usuales, tales como el planteo de cuestiones para discutir en clase, comparar experiencias nuevas con conocimientos ya sabidos, presentar y escuchar diversos puntos de vista ante determinado hecho, proponer y examinar en trabajo grupal los diversos diseños que pueden emplearse para resolver un problema o realizar un experimento, etc.

Las estrategias recién citadas suelen recomendarse para apuntar al desarrollo de capacidades argumentativas en los escolares, que es un rasgo deseable de la educación en ciencias 
La argumentación en la comunidad ...

(KUBLI, 2005; JIMÉNEZ y DÍAZ, 2003, entre otros). Su implementación necesita de docentes que cuenten con cierta experiencia en la participación en debates, enmarcada en saberes teóricos sobre argumentación que posibiliten la reflexión sobre la práctica argumentativa.

Por razones de espacio, las posturas teóricas sobre argumentación no se desarrollan en este artículo (en otros trabajos, ej.: ISLAS, 2006, constan consideraciones sobre las mismas); aquí solamente se atiende a las prácticas argumentativas que pueden introducirse en la formación docente. Se asume que la difusión de ejemplos sobre los debates científicos favorece la reflexión sobre las características de la construcción de conocimiento en ciencias, con miras a que los resultados de esa reflexión puedan operar como estímulo para realizar lo propio en los diversos escenarios de la formación de profesores de ciencias.

\section{Argumentaciones en el seno de una comunidad de investigadores}

Comenzaremos por poner el foco en las modalidades que adopta la comunicación dentro de una comunidad de investigadores, y en su relevancia para el crecimiento académico de los mismos. Se trata de hechos que son bien conocidos por todo integrante de una comunidad científica, aunque tienen poca difusión hacia fuera de ella (BROWN, 2006) y, en particular, entre los profesores de ciencia (KIPNIS, 2001; VIANNA y PESSOA, 2000). Luego de consignar sucintamente aportes de la investigación concernientes a estos temas, presentaremos algunas características detectadas en los intercambios escritos entre investigadores.

\section{Unas notas sobre el estudio de estas cuestiones}

El sociólogo R. Merton se destacó a mediados del siglo XX al impulsar una primera corriente de investigación sobre las características de la ciencia como institución y como tradición (VESSURI, 1991), es decir, el estudio de lo que se da en llamar la sociología interna de la ciencia. Diversas alternativas a esta corriente han ido surgiendo posteriormente y, si bien hoy es éste un campo de interesantes polémicas respecto del valor epistémico del consenso, su examen detallado queda fuera del marco del presente artículo.

Las preocupaciones por la dimensión social del conocimiento científico son abordadas tanto por la filosofía de la ciencia como por otras disciplinas (LONGINO, 2006). La diversidad de enfoques puede advertirse con una mirada a algunos de los temas que conforman los estudios contemporáneos sobre la dinámica interna de la comunidad de investigadores: el rol de la creatividad en ciencias (DUNBAR, 1999), las estrategias cognitivas que emplean los investigadores (NERSESSIAN, 1992), los modos en que está reglada la circulación de la información (CAMPANARIO, 2004a), el tipo de inferencias que operan en los razonamientos durante reuniones de grupo (DUNBAR, 2000), la manera en que se configuran los sistemas de cognición distribuida (GIERE, 2002), los sistemas cognitivo-culturales (NERSESSIAN, 2006). La amplitud del espectro de estudios sobre el proceso de generación de conocimiento científico puede tomarse, en sí misma, como un primer indicador de la complejidad de ese proceso.

Lejos de esta complejidad están las concepciones simplistas (individualistas) de la ciencia que suponen que este proceso sigue un sencillo algoritmo practicado en soledad por 
cada científico cuando se enfrenta a la resolución de un problema. Estas imágenes, sacadas a la luz por la investigación desde hace décadas, mantienen su vigencia, en algunos casos acentuada por la forma en que se presenta a los científicos en los medios de comunicación y también en ocasiones reforzada por la acción (o por la omisión) que se da a este respecto en las prácticas escolares (RAHM, 2007; REIS y GALVÃO, 2005). Para atenuar la impronta del estereotipo del científico aislado, los profesores de ciencias necesitarían contar con ocasiones de acercamiento a la labor científica, una de cuyas características se analiza en este apartado.

\section{La actividad comunicacional de los científicos}

"La comunicación es esencial a la naturaleza y práctica de la ciencia. El hecho de que el número de publicaciones y artículos se haya utilizado como indicador del crecimiento científico establece la producción de publicaciones del proceso de investigación como medida válida de su actividad" (RUSSEL, 2001, p. 4). El contenido de estas afirmaciones es conocido - explícita o implícitamente - por todo investigador; desde los comienzos de su carrera, sabe que cada segmento de su trabajo de investigación (desde la revisión de antecedentes hasta el análisis de conclusiones) ha de ser susceptible de tomar un formato escrito que permita ponerlo a consideración de expertos, con miras a publicarlo. La reputación académica, el renombre, y otras formas de reconocimiento profesional toman para el científico un valor mucho mayor que en otras profesiones, siendo el juicio de sus pares la instancia definitoria (BECHER, 2001). Y para convencer a sus colegas respecto de la validez de sus hallazgos, es preciso que sepa valerse de argumentos explícitamente justificados (KUHN, 1993), además de consideraciones de otros órdenes que le permiten mantenerse en el encuadre que es propio de su comunidad.

Cada comunidad se desenvuelve dentro de ciertos cánones que son aceptados por sus miembros, y que constituyen pautas referidas a tópicos tales como: la extensión de un trabajo a presentar en un congreso o a publicar en una revista, la agenda temática del momento histórico de su disciplina, las metodologías aceptadas como válidas, la cantidad de autores que figuran en un trabajo, los estilos gramaticales y de redacción que se emplean, la cantidad y calidad de referencias bibliográficas, los datos a tener en cuenta para valorar una publicación realizada por otros, etc.

También existen pautas que suelen permanecer tácitas, y ellas forman parte del tema que es de mayor interés en este trabajo: las formas de argumentar, esto es, el uso de estrategias verbales para defender una idea propia y/o para replicar otra ajena, con la intención de arribar a un acuerdo (VAN EEMEREN y GROOTENDORST, 2002). No toda forma de argumentación es aceptada en un determinado campo científico.

Cuando la argumentación científica se toma como objeto de estudio, el encuentro con los datos empíricos puede convertirse en una tarea ardua. Los intercambios argumentativos sincrónicos que acontecen, por ejemplo, en reuniones de equipos de científicos, o en ciertas sesiones de congresos suelen quedar ocultos (sólo accesibles a una investigación etnográfica como la de DUNBAR, 2000). Pero es posible acceder a otros intercambios (diacrónicos, mediados por la reflexión que exige la expresión escrita y los condicionamientos de publicación) en determinadas secciones de las revistas científicas, y en publicaciones específicamente dedicadas a la discusión de diseños de investigación y de sus resultados. 
La argumentación en la comunidad ...

\section{La búsqueda de datos sobre discusiones entre investigadores en Física}

La fuente de datos para este trabajo está conformada por discusiones entre investigadores en Física que se encuentran publicadas en fuentes como las recién mencionadas. La comunidad de físicos dispone, desde hace más de una década, de sitios Web (ej. http:/ /arxiv.org), repositorios de pre-prints, donde pueden publicarse informes de investigación que están en proceso de revisión, así como comentarios de los lectores. También existen revistas electrónicas (ej.: Physical Review Letters) asiduamente empleadas por investigadores, cuyos editores destinan espacios para publicar artículos denominados Comentarios (Comments) en los cuales se presentan objeciones a lo publicado en otros artículos. En las revistas tradicionales, la frecuencia con la que aparecen los Comentarios es baja (ronda el uno por ciento de los artículos); mientras que, por ejemplo, en la revista virtual que editan Koop y Pöschl (2006), el aporte promedio es para uno de cada cuatro trabajos publicados.

La creciente difusión de servicios informáticos ha generado este cambio y otros, igualmente notables, en la circulación de saberes. Aunque "en el clima académico reinante actualmente, el prestigio asociado al hecho de publicar en costosas revistas de papel sometidas a la revisión de los pares es un hecho que los académicos no pueden ignorar" (RUSSEL, 2001, p. 6), la necesidad de mantenerse visible en la comunidad epistémica no es a veces compatible con los tiempos que demora la publicación en revistas impresas. Ello mueve hacia la publicación electrónica, cuyo sistema de revisión es todavía objeto de controversias. Por ello se hace menester la búsqueda de consenso en torno a nuevas normativas que garanticen no sólo la calidad del material que se publica, sino también ciertos recaudos de protección a los autores, tales como: las vías para evitar el plagio, la confiabilidad en la manera de ser citado por otros, etc. (RUSSEL, 2001), es decir, todo aquello ya instalado en el sistema de publicación de impresos en papel, que reposa en 'siglos de tradición' (SCHIRMBACHER, 2006) y que ahora debe ser modificado para las nuevas formas de comunicación entre investigadores. Las inquietudes respecto de este tema han dado lugar a encuentros específicos, artículos en revistas especializadas, y también a un debate asentado en el sitio Web de la conocida revista Nature en el año $2006^{4}$, que recogió opiniones de científicos expertos, con la intención de poner el sistema de revisión por pares a tono con las nuevas demandas (además de analizar otras cuestiones sobre este sistema).

El párrafo anterior, por un lado, ejemplifica el aumento que registran los intercambios de ideas científicas tanto en su publicación como en la facilidad de acceso a los mismos; y por otro lado, muestra a la comunicación científica por vía electrónica como un campo complejo de investigación, en cuyo seno es preciso desarrollar variados estudios (SCHIRMBACHER, 2006; MOREIRA, 2005).

Para este estudio se han tomado algunos de los artículos electrónicos rotulados como Comentario (Comment) y Respuesta (Reply) que contienen, respectivamente, aserciones

\footnotetext{
${ }^{4}$ Algunas de las aportaciones a este debate figuran en las referencias bibliográficas.
} 
de un investigador que cuestionan a las de un autor, y la respuesta de este último. La lectura de estos artículos, y del trabajo original que suscitó la polémica proporciona datos para indagar sobre la forma que toman estas discusiones científicas. En lo que sigue, se expondrá escuetamente la investigación, para lo cual es importante recalcar la delimitación que se acaba de reseñar: se trata de argumentaciones diferentes de las que pueden desarrollarse "en vivo" en una reunión de equipo o en una sesión de un congreso, pues las que aquí se estudian se hallan mediadas por la tarea de los árbitros de las revistas, son el resultado de las reflexiones que realiza un autor antes de enviar un material a publicar, en suma, están condicionadas por los cánones de publicación.

Para seleccionar el material a analizar se tomó, en primer lugar, un criterio de viabilidad, que llevó a la decisión de tomar temas de Óptica. Luego se consultó a investigadores en esta división de la Física, quienes sugirieron una franja temática en la cual abunda el debate: interacción del láser con la materia, e índices de refracción negativos, y un lapso (a partir de enero 2006). En este campo se analizan discusiones publicadas en 26 revistas especializadas que se encuentran disponibles on line para investigadores universitarios en Física. Los artículos objeto de estudio tienen variados estilos, ya que son informes de experimentos, de desarrollos teóricos que los explican, de desarrollos teóricos que predicen resultados factibles de lograr cuando el avance tecnológico lo permita, de simulaciones informáticas de fenómenos físicos, etc.

La lectura de cada terna de artículos (informe-comentario-respuesta) se realizó centrando la atención en las estrategias argumentativas que allí operan. En el apartado que sigue se informa sobre los ejemplos hallados.

\section{Los ejemplos de argumentación entre investigadores en Física}

Tal como se ha dicho, esta investigación no tiene como propósito extraer conclusiones generales respecto de las formas de discusión de los científicos, sino contar con datos empíricos que permitan ejemplificar los razonamientos que se ponen en juego en determinado tipo de debates científicos. En particular interesa mostrar que existen casos en que la validación de un enunciado conclusivo no está fundada en la sintaxis de la inferencia (como es el caso de la deducción) sino que se apela a formas de razonamiento cuyas conclusiones han de validarse a partir de la consideración semántica de los enunciados.

Los debates giran en torno a temas tales como:

1) los límites de validez que se están tomando para un principio/ley/resultado (ej.: "puede aplicarse en este caso, pues los valores de tal variable están dentro de tales márgenes";

2) la pertinencia del empleo de una analogía (ej.: "en esta situación experimental pueden aplicarse los resultados obtenidos en tal trabajo, ya que..."); de un formalismo matemático (ej.: “dado que se está en un régimen de saturación, la ecuación que corresponde usar es..."); de un diseño experimental (ej.: "la respuesta del artefacto puede ser cuantificada usando el dispositivo...”);

3) la modelización efectuada (ej.: "consideración fotónica de la luz, versus consideración ondulatoria");

4) los márgenes de incerteza con los cuales se realizan los cálculos, sean estos experimentales o teóricos; 
5) cuál es el registro experimental que justifica una interpretación (ej.: "los autores deberían haber advertido que esas líneas no necesariamente son provocadas por la doble fotoionización, sino que pueden deberse a...”).

Aunque el número de casos analizados no habilita para formular conclusiones generales, en los razonamientos que se infieren a partir de los debates analizados, las analogías y abducciones aparecen con las siguientes características:

. Es frecuente el empleo de inferencias analógicas. Como es sabido, de ellas no pueden extraerse conclusiones que la lógica valide, sino que ofrecen pautas para orientar la interpretación de hechos, como en este caso son los registros experimentales, los resultados de desarrollos teóricos, etc. La analogía se utiliza, por ejemplo, cada vez que se compara el experimento del cual se está hablando con otro registro experimental que ya ha sido "validado" por la comunidad. Esta comparación se halla tanto en los Comentarios analizados, como en las Respuestas a los mismos y en los artículos originales.

- Las controversias nacen por desacuerdos respecto de cuáles son los atributos que cada científico (el autor y el comentador) aprecia como esenciales de las situaciones bajo análisis. Por ejemplo: ciertas influencias espurias generadas por el instrumental, consideradas despreciables por los autores de un trabajo, son entendidas por los comentadores como decisivas en la configuración de la situación y, en consecuencia, en la interpretación de resultados.

. La mayoría de los razonamientos que conforman la línea cardinal de cada argumentación han podido ser formalizados como abducciones. Esto es, ante un hecho no explicado (ej.: "aparición de ciertas señales en una imagen"), se apela a una regla general conocida (en este caso: "el fenómeno X da lugar a esas señales") y la conclusión reviste un carácter hipotético (en este ejemplo: "las señales permiten suponer que ha ocurrido el fenómeno X"). ${ }^{5}$ Esta conclusión ha de ser sometida luego a contrastaciones, sean estas de tipo empírico o de control de su consistencia con las teorías consensuadas y/o con otros experimentos ya validados.

Se han encontrado Comentarios que cuestionan la conclusión debido a que apelan a otra regla ("el fenómeno Z da lugar a esas señales"), que es aceptada como válida para el tema en cuestión, lo cual origina una interpretación diferente de los hallazgos empíricos. En términos semióticos, se diría que ante un mismo signo (las señales en la imagen) hay más de una interpretación aceptable. La discusión entre pares conduce hacia la determinación de cuál de esas interpretaciones es la más adecuada, con lo cual, además, se evidencia la capacidad de autocorrección de la ciencia (MARAFIOTI, 2004).

Algo similar ocurre en el territorio de los estudios teóricos, cuando existe la posibilidad de más de un encuadre para un cierto tópico.

Es importante recordar que los resultados recién reseñados conciernen a una labor que se lleva a cabo en los espacios de las universidades; espacios que son también el escenario para la formación de los futuros docentes de ciencias. Empero, la distancia de estos estudiantes con estos hechos parece ser notable, pues cabe preguntarse: ¿cuánto sabe de comunicación entre científicos un profesor de ciencias? ¿Los intercambios de ideas científicas son analizados

${ }^{5}$ El ejemplo corresponde a: "Measurements of Energetic Proton Transport through Magnetized Plasma from Intense Laser Interactions with Solids", de Clark et al. (2000), comentado por Gaillard et al. (2006). 
en las Epistemologías que se estudian en los Profesorados y en la formación profesional? La investigación sobre nociones de ciencia en docentes (ej. BARONA et al., 2004) arroja resultados que sugieren respuestas negativas a estas preguntas.

La intención del trabajo presentado en este artículo es contribuir a la reflexión sobre posibles maneras de utilizar los datos obtenidos para mejorar la formación epistemológica de los profesores.

\section{Argumentaciones en la formación de profesores de física}

Si bien forman parte de la comunidad universitaria, los estudiantes de Profesorado raramente están al tanto de las actividades de investigación que llevan a cabo sus propios profesores, y algo semejante se da en los cursos de capacitación docente. Por ejemplo: Vianna y Pessoa (2000, p. 35), realizando cursos con docentes en servicio, encuentran que "los profesores cursantes, en su gran mayoría, no han tenido oportunidad, durante su formación académica y profesional, de conocer el proceso de la producción científica". Similares observaciones se han registrado en nuestra Facultad cuando estudiantes y graduados de Profesorado en Física, en ocasión de participar de cursos de actualización dictados por investigadores en Física, manifestaron que para la mayoría de ellos, ésa era la primera vez que ingresaban a laboratorios de los Institutos de investigación localizados en el mismo Campus en el cual cursaran su carrera docente. En un trabajo con futuros profesores de Física (ISLAS y PESA, 2002) se constató que ellos desconocían ciertas prácticas propias de la investigación científica, en ese caso, la elaboración y empleo de modelos. En ese trabajo se entrevistó también a científicos que, siendo profesores de esos estudiantes, reconocían al modelado como una parte esencial de su tarea de investigación.

Se advierte, entonces, que el contacto investigador-estudiante no sería suficiente para que los futuros docentes estén al tanto de ciertos atributos que caracterizan a la tarea de investigación científica.

La investigación sobre concepciones epistemológicas de docentes (en formación y en servicio) viene mostrando que muchos de ellos adhieren a las visiones simplistas de la ciencia (detalles sobre ellas pueden encontrarse, por ejemplo, en REIS y GALVÃO, 2005). Estas visiones excluyen del campo científico la duda, el disenso, y toda forma de argumentación y de diálogo (ISLAS, STIPCICH y DOMÍNGUEZ, 2006); las aseveraciones de la ciencia parecen ser validadas en una de dos maneras (que son, por cierto, incompatibles entre sî): por la vía inductiva, a la manera del empirismo, o bien por la Lógica pura, prescindiendo de los componentes semánticos de los enunciados.

Subsiste la creencia según la cual tanto un desarrollo teórico como un experimento siempre conducen a un resultado que admite una interpretación única y, por lo tanto, indiscutible. El rol del científico parece estar reducido a "leer correctamente" lo que la naturaleza exhibe ante sus ojos (aunque en nuestros días, la mediación de instrumentos sea relevante), y con ello se prescinde de las sucesivas semiosis que tienen lugar cada vez que se interpretan resultados.

$\mathrm{Al}$ reconocer que estas nociones de ciencia subyacen en las decisiones que se toman en el aula, se comprende por qué es frecuente que los profesores presenten en sus clases al 
contenido científico como una entidad absoluta, no susceptible de críticas y - menos aún - de cambios. Una ligera reflexión bastaría para percatarse de que con esto quedarían cerradas las oportunidades de progreso, y es ésta una tarea mínima que habría de concretarse en la formación de los docentes.

A lo anterior se suma que puede ser difícil para un profesor admitir que no se halla en posesión de verdades incuestionables. Tal como consigna Kubli (2005), es difundida la concepción de la clase como escenario en el cual el docente presenta solamente saberes establecidos, y nunca enfrenta a sus alumnos con situaciones dilemáticas, que fomenten el debate. La ciencia queda presentada como una mera retórica de conclusiones (PRAIA y CACHAPUZ, 2002), pues no se hace referencia a la actividad que se da en su seno; esto también ayuda para que la clase quede configurada como un ámbito donde priman las certezas.

Pero es sabido que la actividad en el aula no es totalmente previsible, no se pueden predecir con exactitud todos sus resultados. Desde esta perspectiva, algunos autores opinan que quizás entre los objetivos de la formación de profesores habría que incluir el de prepararlos para manejarse en situaciones de incertidumbre (FELDMAN, 2004). Esta consideración general respecto de la formación docente cobra más relevancia si se piensa en una disciplina como la Física, donde la creencia en la inmutabilidad de las construcciones teóricas y en la fijeza de las metodologías abona la suposición de que los debates no existen en el seno disciplinar. Las concepciones epistemológicas que entran en juego aquí habrían de ser objeto de análisis en los cursos de formación docente.

\section{Algunas acciones posibles}

En trabajos anteriores (ej. ISLAS, SGRO y PESA, 2005) hemos informado que la inclusión de la Epistemología en los Planes de estudio de los Profesorados en Física (en las Universidades Nacionales de Argentina) ha ido creciendo en los últimos años, resultado alentador en lo que hace a la problemática que nos ocupa. Además, se registra la presencia de Epistemologías no standard en los Programas de las asignaturas que han sido revisados. Si bien se ha encontrado diversidad en cuanto a las líneas que se estudian en cada caso, en todos ellos está presente la Epistemología de Kuhn. La noción kuhniana de paradigma es un concepto básicamente sociológico, y su estudio involucra, además de textos y prácticas científicas, a las publicaciones, las reuniones, y otras actividades que vehiculizan la comunicación entre los miembros de cada comunidad (HELLER, 2005). La noción de paradigma es una, entre otras, de las que hacen posible adentrarse en consideraciones sobre la dinámica interna de la ciencia.

Del mismo modo, la valoración del error y de los obstáculos epistemológicos que realiza Bachelard, la no-uniformidad de metodologías propuesta por Feyerabend, o las disciplinas profesionales en el sentido en que las analiza Toulmin, configuran umbrales de acceso a la sociología interna de la ciencia que, una vez traspuestos, pueden facilitar la comprensión de cómo se organiza, funciona y evoluciona esa sociedad específica que es la conformada por los científicos en actividad.

Se podría estimular la superación de los límites que imponen a los docentes sus concepciones simplistas de la ciencia, adoptando lo que se da en llamar un 'giro hacia una visión más cultural de la tarea científica' (TOBIN, 2006), que incluya la reflexión sobre la Historia, la 
Filosofía y la Sociología de la ciencia (WANG y SCHMIDT, 2001), como complemento de los temas abordados en Epistemología. El estudio de estos temas puede hacer interesantes aportes teóricos para lograr que los profesores estén en mejores condiciones de acercar a sus alumnos a la comprensión de los hechos científicos desde variadas dimensiones.

Ahora bien, estos cambios en la selección de ideas esenciales de Epistemología y/o incrementos en los temas a tratar en los cursos (ya sean cátedras de los Profesorados o formen parte de programas de capacitación) se vislumbran como viables, pero significan una solución meramente parcial si lo que se intenta es formar un docente capacitado para presentar a sus alumnos una visión de la ciencia que contemple aspectos como los hallados en la investigación que brevemente se ha relatado en este artículo. Para apreciar la potencia de la práctica argumentativa en la construcción de conocimiento será preciso acompañar la modificación de contenidos con ciertos cambios en la dinámica de las clases de estos cursos que, sin ánimo de emular las discusiones científicas, estimulen una postura más crítica respecto de la manera en que se abordan los contenidos (CAMPANARIO, 2004b). El espectro de actividades de aula se podría ampliar si se acrecienta la variedad y las ocasiones de debate en las clases. Los ámbitos más propicios para ello podrían ser, además de los cursos de Epistemología, los dirigidos a las problemáticas más específicas de la formación para el aula, tales como las Didácticas y la Práctica de la Enseñanza, así como los seminarios dedicados a la discusión de temas específicos.

Los debates en torno a las posibles maneras de llevar a cabo una propuesta didáctica, de seleccionar un libro de texto, de interpretar lo que se ha observado en una clase, etc. pueden ser, para el docente en formación, ocasiones para familiarizarse con el ejercicio de las capacidades que demanda la participación activa en discusiones que buscan lograr un acuerdo (VAN EEMEREN y GROOTENDORST, 2002), y con ello mejorar su preparación para que en su tarea docente valorice a la crítica como instrumento pedagógico (LABURÚ, ARRUDA e NARDI, 1998) promotor de la creación de nuevos saberes. En este sentido, la propia formación pedagógica puede privilegiar una metodología dialógica que sin duda ayudaría a una visión más dinámica no sólo de la producción de conocimiento, sino también de la acción educativa que es responsable de la transmisión de saberes específicos y de la forma, más general, de procesarlos.

Finalmente, cabe tener en cuenta que la mayoría de las cátedras universitarias de Profesorado están a cargo de investigadores, y que algunos de ellos participan de las acciones de capacitación docente en servicio. Sería conveniente estimular el acercamiento de los estudiantes a la tarea de investigación que sus propios profesores realizan, reduciendo la distancia que parece existir entre la tarea docente y la de investigación. Quienes hacemos investigación en educación científica al tiempo que actuamos como formadores de docentes disponemos de múltiples ocasiones para dialogar con ellos acerca de cómo se desarrolla la tarea de investigación. Tal como lo consigna Campanario (2004b), la comunicación con colegas es un factor de notable influencia en la actividad de todo investigador, pues el éxito o el fracaso de su trabajo están siempre supeditados a la opinión de sus pares. Sin embargo, "éste es uno de los elementos a los que menos atención se presta en filosofía de la ciencia y, como consecuencia, en la presentación que hacemos de la ciencia al alumnado y en las visiones que transmitimos" (CAMPANARIO, 2004b, p. 366). La faceta de investigador que tiene la mayoría de los integrantes del cuerpo de formadores de docentes queda frecuentemente oculta para sus alumnos; se los priva, de este modo, de sostener intercambios de ideas sobre la ciencia en su propia aula y con 
científicos conocidos, aprovechando que disponen naturalmente de espacios que muchos docentes (como los que trabajan con RAHM, 2007) deben gestionar invitando a investigadores foráneos para que dialoguen con los alumnos.

\section{Comentario final}

Las concepciones epistemológicas de los docentes tienen, entre sus componentes, una visión acerca de la dinámica interna de la ciencia que no se condice con lo que en la vida cotidiana realizan los científicos. Habituados a estudiar y enseñar en torno a asuntos consensuados, los docentes dejan de lado el proceso de producción del conocimiento, minimizando sus rasgos sociales, uno de los cuales es el de las controversias internas y los debates respecto de cuál de todas las posibles interpretaciones de un fenómeno es la más ajustada para explicarlo.

Un docente compenetrado en estos temas podría ayudar a sus alumnos a comprender significativamente no sólo la información científica, sino también la trama social dentro de la cual ella se genera y desarrolla (WANG y SCHMIDT, 2001). Pero para ello, ese docente debería tener durante su formación oportunidades de conocer más de cerca esa trama social de la ciencia y más aun, podría advertir cierta resonancia entre los modos de discutir sobre saberes pedagógicos y las discusiones sobre conocimientos científicos.

\section{Referencias}

BARONA R., C. et al. La concepción de la naturaleza de la ciencia (CNC) de un grupo de docentes inmersos en un programa de formación profesional en ciencias. Revista

Electrónica de Investigación Educativa, México, v. 6, n. 2, 2004. Disponible en: <http:/ /redie.uabc.mx/vol6no2/contenido-barona.html>. Acceso en: 17 oct. 2007.

BECHER, T. Tribus y territorios académicos. Barcelona: Gedisa, 2001.

BROWN, T. Perspective: 'I don't know what to believe'. Nature. 2006. Disponible en: <http://www.nature.com/nature/peerreview/debate/nature04988.html>. Acceso en: 17 oct. 2007.

CAMPANARIO, J. Científicos que cuestionan los paradigmas dominantes: algunas implicaciones para la enseñanza de las ciencias. Revista Electrónica de Enseñanza de las Ciencias, v. 3, n. 2, 2004a. Disponible en: <http://www.saum.uvigo.es/reec>. Acceso en: 17 oct. 2007.

Algunas posibilidades del artículo de investigación como recurso didáctico orientado a cuestionar ideas inadecuadas sobre la ciencia. Enseñanza de las Ciencias, Barcelona, v. 22, n. 3, p. 365-78, 2004b. 
Islas, S. M.; Sgro, M. R.; Pesa, M. A.

CLARK, E. et al. Measurements of energetic proton transport through magnetized plasma from intense laser interactions with solids. Physical Review Letters, Nova York, v. 84, n. 4, p. 670-3, 2000.

DUNBAR, K. How scientists think in the real world: implications for science education. Journal of Applied Developmental Psychology, Washington, v. 21, n.1, p. 49-58, 2000.

Scientific creativity. Encyclopedia of creativity. Canada, 1999. v. 1.

FELDMAN, D. Ayudar a enseñar. Buenos Aires: Aique, 2004.

GAILLARD, S. et al. Comment on "Measurements of energetic proton transport through magnetized plasma from intense laser interactions with solids". Physical Review Letters, Nova York, v. 96, n. 24, 2006.

GIERE, R. Distributed cognition in epistemic cultures. Philosophy of Science, Chicago, v. 69, n. 2, p. 632-44, 2002.

HELLER, M. Ciencia incierta: la producción social del conocimiento. Buenos Aires: Biblos, 2005.

ISLAS, S. Los debates en las clases de Física. In: CONGRESO NACIONAL "LEER, ESCRIBIR Y HABLAR HOY LA CIENCIA, LA LITERATURA, LA PRENSA”, 1., 2006, Buenos Aires. Memorias... Buenos Aires: Universidad Nacional del Centro de la Provincia de Buenos Aires, 2006. 1 cd-rom.

ISLAS, S.; PESA, M. ¿Qué ideas tienen los profesores de Física del nivel medio respecto del modelado?.Ciência \& Educação, Bauru, v. 8, n 1, p. 13-26, 2002.

ISLAS, S.; SGRO, M.; PESA, M. La comunicación entre científicos: un tema de interés para la formación de profesores de Física. In: JORNADAS DE INVESTIGACIÓN

EDUCATIVA, 4., 2005, Córdoba. Memorias... Córdoba: Facultad de Filosofía y

Humanidades, Universidad Nacional de Córdoba, 2005. 1 cd-rom.

ISLAS, S.; STIPCICH, S.; DOMÍNGUEZ, A. El lugar de la argumentación en la formación de profesores de ciencias. Revista Chilena de Educación Científica, Santiago de Chile, v. 5, n. 1, p. 67-74, 2006.

JIMÉNEZ A., M. P.; DÍAZ DE BUSTAMANTE, J. Discurso de aula y argumentación en la clase de ciencias: cuestiones teóricas y metodológicas. Enseñanza de las Ciencias, Barcelona, v. 21, n.3, p. 359-70, 2003.

KIPNIS, N. Scientific controversies in teaching science: the case of Volta. Science \& Education, Netherlands, v. 10, n. 1, p. 33-49, 2001.

KOOP, T.; PÖSCHL, U. Systems: an open, two-stage peer-review journal. Nature, 2006. Disponible en: < http://www.nature.com/nature/peerreview/debate/nature04988.html> Acceso en: 17 oct. 2007. 
La argumentación en la comunidad ...

KUHN, D. Science as argument: implications for teaching and learning scientific thinking. Science Education, Nova York, v. 77, n. 3, p. 319-37, 1993.

KUBLI, F. Science teaching as a dialogue - Bakthin, Vygotsky and some applications in the Classroom. Science \& Education, Netherlands, v. 14, n. 6, p. 501-34, 2005.

LONGINO, H. The social dimensions of scientific knowledge. Stanford: Stanford Encyclopedia of Phylosopy, 2006. Disponible en: < http://plato.stanford.edu/entries / scientific-knowledge-social/>. Acceso en: 7 nov. 2007.

LABURÚ, C.; ARRUDA, S.; NARDI, R. Os programas de pesquisa de Lakatos: uma leitura para o entendimento da construção do conhecimento em sala de aula em situações de contradição e controvérsia. Ciência \& Educação, Bauru, v. 5, n. 2, p. 23-38, 1998.

MARAFIOTI, R. Charles S. Peirce: el éxtasis de los signos. Buenos Aires: Biblos, 2004.

MOREIRA, W. Os colégios virtuais e a nova configuração da comunicação científica.

Ciência da Informação, Brasília, v. 34, n. 1, p. 57-63, 2005.

NERSESSIAN, N. The cognitive-cultural systems of the research laboratory. Organization Studies, Minnesota, v. 27, n. 1, p. 125-45, 2006.

How do scientists think? Capturing the dynamic of conceptual change in science. In: GIERE, R. (Eds.). Cognitive models of Science. Minnesota: University of Minnesota Press, 1992. p. 3-45.

PRAIA, J.; CACHAPUZ, A. Problema, teoria e observação em ciência: para una reorientação epistemológica da educação em ciência. Ciência \& Educação, Bauru, v. 8, n. 1, p. 127-45, 2002.

RAHM, J. Youths' and scientists' authoring of and positioning within science and scientists' work. Cultural Studies in Science Education, Nova York, v. 1, n. 3, p. 517-44, 2007.

REIS, P.; GALVÃO, C. Controvérsias sócio-científicas e prática pedagógica de jovens professores. Investigações em Ensino de Ciencias, Porto Alegre, v. 10, n. 2, 2005. Disponible en: <http://www.if.ufrgs.br/public/ensino/revista.htm>. Acceso en: 7 nov. 2007.

RUSSELL, J., La comunicación científica a comienzos del siglo XXI. Revista Internacional de Ciencias Sociales, La Rioja, n. 168, 2001. Disponible em: < http:// www.oei.es/salactsi>. Acceso en: 7 nov. 2007.

SCHIRMBACHER, P. The new culture of electronic publishing. Ciência da Informação, Brasília, v. 35, n. 2, p. 51-7, 2006.

TOBIN, K. Toward a cultural turn in science education. Cultural Studies of Science Education, Netherlands, v. 1, n. 1, p. 7-10, 2006. Disponible en: <http:// www.springerlink.com/content/vu4504873757/>. Acceso en: 22 jul. 2007. 
Islas, S. M.; Sgro, M. R.; Pesa, M. A.

VAN EEMEREN, F; GROOTENDORST, R. Argumentación, comunicación y falacias. Chile: Universidad Católica de Chile, 2002.

VESSURI, H. Perspectivas recientes en el estudio social de la ciencia. Interciencia, Venezuela, v. 16, n. 2, p. 60-8, 1991.

VIANNA, D.; PESSOA, A. Formação permanente: a necessidade da interação entre a ciência dos cientistas e a ciência da sala de aula. Ciência $\boldsymbol{\&}$ Educação, Bauru, v. 6, n. 1, p. 31-42, 2000.

WANG, H.; SCHMIDT, W. History, philosophy and sociology of science in science education: results from the third international mathematics and science study. Science $\boldsymbol{\&}$ Education, Netherlands, v. 10, p. 51-70, 2001.

Artigo recebido em dezembro de 2008 e aceito em junho de 2009. 